\title{
Epilepsy and Anesthesia
}

\author{
Marcius Vinícius Mulatinho Maranhão, TSA ${ }^{1}$, Eni Araújo Gomes ${ }^{2}$, Priscila Evaristo de Carvalho ${ }^{2}$
}

Summary: Maranhão MVM, Gomes EA, Carvalho EP - Epilepsy and Anesthesia.

Background and objectives: Epilepsy is one of the most frequent chronic neurological diseases. Although anesthesia for epilepsy patients is more common in neurosurgery, this group of patients needs, just as the general population, anesthesia for different diagnostic and therapeutic procedures. This article aims to address the issues of greatest interest to the anesthesiologist in the perioperative management of epileptic patients undergoing anesthesia for non-neurosurgical procedures.

Content: We discuss relevant aspects of pathophysiology, classification and diagnosis of epilepsy; anticonvulsant therapy and interactions with anesthetic drugs; surgery and the ketogenic diet; pro-and anticonvulsant effects of drugs used in anesthesia; preoperative evaluation, intra- and postoperative conduct in epileptic patients, as well as the diagnosis and treatment of perioperative seizures.

Conclusions: In the perioperative management of epileptic patients is important for anesthesiologists to identify the type of epilepsy, the frequency, severity and the factors triggering the epileptogenic crises; the use of anticonvulsant drugs and possible interactions with drugs used in anesthesia; the presence of ketogenic diet and stimulatory of the vagus nerve, and its implications in anesthetic techniques. It is essential the understanding of pro- and anticonvulsant properties of drugs used in anesthesia, minimizing the risk of seizure activity in the intra- and postoperative. Finally, it is important to outline the diagnosis and initiate treatment of seizures, perioperative, which offers lower both morbidity and mortality.

Keywords: Epilepsy; Anesthesia; Perioperative care; Seizures.

[Rev Bras Anestesiol 2011;61(2): 232-254] CElsevier Editora Ltda.

\section{INTRODUCTION}

Epilepsy is a chronic neurological disorder and may be progressive in relation to cognitive impairment, frequency and severity of critical events characterized by recurrent seizures ${ }^{1}$.

Epilepsy is as old as mankind. It was first described about 3,000 years ago in Akkadian, Mesopotamia (Iraq). The seizures were attributed to the god of the moon. In the early $17^{\text {th }}$ century, William Gilbert described the electrical phenomena responsible for epilepsy, discarding the mystical and supernatural theory. The word epilepsy is derived from the Greek verb "ëpilamvanein" (attack, seizure) ${ }^{2}$.

Epilepsy is one of the most frequent neurological diseases, only surpassed by strokes ${ }^{3}$. It affects about $1 \%$ of world population. The incidence of this disease varies with age, sex, race, type of epilepsy syndrome and socioeconomic conditions. In developed countries, the prevalence of epilepsy is around $0.5 \%$ of the population. In developing countries, it ranges from $1.5 \%$ to $2 \%$ of the population. In the U.S. there are two million people with epilepsy and 300,000 are children under the age

Received from the Discipline of Pharmacology, Instituto de Ciências Biológicas (IBC), Universidade de Pernambuco (UPE) - Anesthesiology Service of the Hospital Universitário Oswaldo Cruz - CET/SBA do Hospital da Restauração, Hospital Getulio Vargas and Hospital Universitário Oswaldo Cruz.

1. Pharmacolgy Professor of the ICB of UPE; Anesthesiologist of the Hospital Universitário Oswaldo Cruz; Cor-responsable for CET/SBA of Hospitals Restauração, Getúlio Vargas and Universitário Oswaldo Cruz; Teaching and Training Commission Member/SBA

2. Undergraduate Medicine Student UPE

Submitted on June 28, 2010.

Approved on October 20,2010.

Correspondence to:

Dr. Marcius Vinícius M. Maranhão

Rua Manuel Bernardes 134/702

Magdalene

50710-350 - Recife, Brazil

E-mail: Gabriel.n@uol.com.br of 14 years ${ }^{4}$. There are few studies on the prevalence and there are no published studies on the incidence of epilepsy in Brazil. It is estimated that in our country 340,000 new cases of epilepsy are diagnosed each year with 1.8 million patients with active epilepsy, and that at least 9 million people have had at seizure some time in life 5,6 . In developed countries, the curve of epilepsy by age shows higher incidence in children and the elderly, and it shows higher incidence in young adults in developing countries, which probably reflects different etiologies. While in developed countries there is a predominance of developmental disorders and idiopathic epilepsy in children (i.e. epilepsy resulting from genetic susceptibility), and the vascular and degenerative processes of aging, in developing countries infectious and parasitic causes, and head trauma contribute to a significant percentage of cases ${ }^{1,4}$. It is more common in males and in lower socioeconomic classes ${ }^{7}$. It is important to emphasize that the presence of epilepsy is high in children with mental retardation, cerebral palsy, autism, psychiatric behavior disorders. Approximately $30 \%$ of children with autism may have different types of seizures during adolescence ${ }^{4}$.

The epileptic patient has a higher mortality from unexpected sudden death, status epilepticus and a high suicide rate ${ }^{4}$. The disease is characterized by a state of hyperactivity of neurons and brain circuits capable of generating synchronous electrical discharges, which may manifest itself in various forms, since interictal encephalographic discharges to prolonged seizures with epileptic crises or, in more severe cases, assuming the form of status epilepticus, a condition characterized by isolated prolonged seizures or by repeated seizures in short intervals. The interictal discharge corresponds, at the cellular level, to synchronized paroxysmal discharges of certain neuronal population, represented by action potential bursts 1 . 


\section{PATHOPHYSIOLOGY}

Excessive and synchronous neuronal discharges that characterize epileptic phenomenon may originate from one point of the cerebral hemisphere (focal seizures) or a more extensive area involving the two hemispheres (generalized seizures). The focal seizures may become secondarily generalized seizures with the spread of the discharge. These excessive and synchronous neuronal discharges are provoked by excitatory stimuli, mediated mainly by glutamate (the major excitatory neurotransmitter) or the lack of inhibition mediated by GABA (gamma aminobutyric acid), an inhibitory neurotransmitter. The generalized seizures involve thalamic circuits in the generation of diffuse, bilateral and synchronous discharges, whereas focal seizures involve part of one or both brain hemispheres. The cell damage and the harmful consequences of generalized seizures arise from the influx of calcium ions during the depolarization and activation of excitatory amino acid receptors, promoting acute cell necrosis and apoptotic cell death in the long term, then confirming the excitotoxic cell damage.

\section{CLASSIFICATION}

Although there are several classifications of epileptic seizures, the clinical classification most used is the proposal by the International League Against Epilepsy (Chart 1) ${ }^{9}$.

Chart 1 - Classification of Epileptic Seizures.

Partial seizure
Simple partial seizure
Complex partial seizure
Generalized seizure
Typical absence seizure
Atypical absence seizure
Myoclonic seizure
Tonic-clonic seizure
Tonic seizure
Clonic seizure
Atonic seizure
Status epilepticus

The focal seizures (partial) are those whose clinical manifestations indicate the involvement of a region of one cerebral hemisphere. With the spread of discharges, the focal seizures may develop into a tonic-clonic seizure. This is what is called a focal crisis with secondary generalization. The focal seizures can be classified as:

Simple partial: in this group, the epileptic phenomenon is represented by auras. Among them, are the sensory (paresthesias, pain and visceral sensations), visual, auditory, olfactory and gustatory crises. Consciousness is preserved ${ }^{1,9}$.

Complex partial: hallucinations consist of multisensory experiences that shape and include affective and perceptual phenomena mnemonics involving delusions and hallucinations. Its quality is similar to everyday experiences, however recognized by the individual as something that occurs outside the realm of the real and, sometimes, lived in an extraordinary manner. The conscience is compromised ${ }^{1,9}$.

In generalized seizures, the clinical manifestations indicate involvement of both hemispheres from the beginning, and electroencephalographic discharges are bilateral. As in generalized seizures, the ascending reticular system is affected by early discharges, and consciousness is always impaired ${ }^{1,9}$. The generalized seizures can be classified as:

- Typical absence seizures (petit mal): it consists of brief episodes of impaired consciousness, accompanied by very mild motor manifestations, such as oral and manual automatisms, blinking, increased or decreased muscle tone and autonomic signs. They last about 10 to 30 seconds, and start and end abruptly, occurring usually several times a day. They are triggered by hyperventilation, activation of such importance that failure to observe the classic crisis during hyperventilation for three to five minutes should cast doubt on that diagnosis. Absences may manifest themselves only with impairment of consciousness, with discrete clonic, tonic or atonic components, with automatisms or autonomic phenomena as incontinence (enuresis), for example ${ }^{1,9}$.

- Atypical absence seizures: in these crises, the impairment of consciousness is decreased, the beginning and ending are less abrubpt and muscle tone is often changed. They are not triggered by hyperpneia ${ }^{1,9}$.

- Myoclonic seizures: they are characterized as sudden, brief, shock-like seizures. They can affect facial or torso muscles, extremities, muscle group or isolated muscles, and can be generalized, occurring isolated or repeatedly. Myoclonic seizures commonly occur after sleep deprivation, waking or sleeping ${ }^{1,9}$.

- Generalized tonic-clonic seizures (grand mal): are characterized by sudden loss of consciousness with tonic and then clonic contractions of the four limbs, apnea (which can lead to hypoxemia), incontinence, sialorrhea and tongue biting, for about a minute. In the phase of tonic contraction, the air can be forced through the closed glottis, resulting in epileptic cry. The post-seizure period is characterized by mental confusion, headache, drowsiness, nausea and muscle pain, symptoms that can be viewed isolated or in association ${ }^{1,9,10}$.

- Tonic clonic and atonic seizures: it may result in loss of consciousness, with tonic or clonic component described above, especially in children. Atonic seizures have also been described ${ }^{9}$.

- Continuous generalized seizures (status epilepticus): Characterized by continuous or repeated seizures without recovery or incomplete recovery of consciousness. Seizures have duration of at least 30 minutes and may extend for 48 hours, with four to five seizures per hour. It is a medical emergency and can be fatal. Mortality can reach $20 \%$. With the progression of seizures, skeletal muscle activity is reduced, 
and seizure activity may be evident only by EEG. Respiratory effects of status epilepticus include inhibition of the respiratory centers, uncoordinated skeletal muscle activity with ventilation impairment. The abnormal autonomic activity can cause bronchospasm. It requires immediate treatment to avoid the risk of brain injury 1,9-12.

\section{DIAGNOSIS}

The diagnosis of epilepsy is based on clinical history, description of seizure activity by those who witnessed, physical examination (looking for focal findings) and encephalographic findings 4 .

Nonspecific changes such as headache, mood changes, lethargy and myoclonic contraction warn some patients to an impending seizure, within hours of its occurrence. These prodromal symptoms are different from aura, which may precede the generalized seizure by a few seconds or minutes, and is part of epileptic seizure. In most patients, seizures occur unpredictably, without any regard to the posture or activity. Occasionally, however, crises occur in certain situations, such as sleep, or related to external predisposing factors such as sleep deprivation, poor diet, stress, menstruation, ingestion or withdrawal of alcohol and drug use. Fever and nonspecific infectious process can trigger seizures in epileptic patients. In a few patients, the seizures can be provoked by specific stimuli such as television flashing (photosensitive epilepsy), music or reading ${ }^{9}$.

Physical examination between seizures in idiopathic epilepsy shows no abnormalities, but in the immediate posictal period, extensor plantar response can be observed ${ }^{9}$.

In partial seizures, differential diagnosis should be performed with transient ischemic attack, aggressiveness and panic attacks ${ }^{9}$. In generalized seizures, differential diagnosis should be done with syncope, cardiac arrhythmias, brainstem ischemia and pseudoepilepsia (due to psychiatric illness) ${ }^{9}$.

Blood tests and lumbar puncture are rarely necessary for the diagnosis of epilepsy, except in the presence of suspected electrolyte abnormalities, liver diseases, cancer, infectious diseases or exposure to toxins, althouhg that conduct is not yet consensus ${ }^{4,9}$. Some authors consider it important, in the initial evaluation, the hemogram, glucose, liver function tests, kidney function and serological tests for syphilis. Serum prolactin and creatinine significantly increased after the tonicclonic seizures in most patients ${ }^{9}$.

A CT scan or MRI is indicated for patients with focal neurological signs and symptoms, focal seizures or EEG findings of a focal seizure; some neurologists routinely indicate imaging for all patients in the initial evaluation of a seizure ${ }^{9}$.

In children, emergency neuroimaging studies are performed when post convulsive deficit or altered mental status occurs that do not disappear quickly. Neuroimaging studies can be performed electively in patients where there is no determined cause for the focal seizure or infants who have a non febrile seizure ${ }^{4}$.
Epileptiform EEG changes are essential in the diagnosis and therapeutic management in the epileptic patient ${ }^{8}$. This is a simple and inexpensive diagnostic method to determine the physiological manifestations of abnormal cortical excitability associated with epilepsy. The EEG shows three types of information: confirmation of abnormal electrical activity, the type of seizures and localization of the convulsive focus. Specificity is high, $78-98 \%$, but sensitivity is low, around $25-26 \%$. This is due to the fact that the EEG analyzes only the superficial layers of the cerebral cortex. The short duration of routine EEG is one of the reasons why only $50 \%$ of the initial EEG shows epileptiform discharges ${ }^{4}$. In general, the EEG is performed after 48 hours or more of the seizure, although more recent evidence reveals that investigations within the first 24 hours may show interictal discharges ${ }^{13}$. Preferably, the EEG should be performed during sleep, light stimulation and hyperventilation, because certain types of seizures occur in these conditions ${ }^{14}$.

Other tests used in diagnosis of epilepsy include video EEG, functional magnetic resonance imaging, positron emission tomography (PET) and single photon emission computed tomography (SPECT).

\section{TREATMENT}

Approximately $10 \%$ of the population has a single convulsive episode during their lifetime. In this situation, the use of anticonvulsants is not indicated. After the occurrence of a second seizure, the diagnosis of epilepsy is confirmed, initiating then the routine treatment with antiepileptic drugs 4 .

The choice of the anticonvulsant should be made according to the type of crisis, the effectiveness and side effects, and used as monotherapy whenever possible, i.e. the goal of pharmacological treatment is to control seizures without side effects ${ }^{1,4,15}$. It is important to emphasize that most of the adverse effects of antiepileptic drugs is dose-dependent, i.e. decreasing the dose of the drug reduces the severity of side effects, with no need to discontinue the drug ${ }^{4}$. The appropriate prescription of anticonvulsants requires knowledge of the pharmacokinetics of the drug. One must consider that, once the pharmacological treatment is established, the anticonvulsant can only achieve metabolic balance after a period of at least four half-lives, the time required to obtain tissue saturation. Monitoring blood levels of antiepileptic drugs is rarely necessary, because blood levels of drugs do not consistently correlate with clinical toxicity or clinical control of seizures. The suspension of antiepileptic drugs can be considered in the absence of seizures after two years of initiation of drug therapy, except for neonates in whom therapy can be discontinued 2 weeks after the last seizure. The risk of recurrence of seizures is high in patients with structural lesion, electroencephalographic abnormalities, seizures onset in adolescence, neurological disorders, severe epilepsy, when they require multiple medications or when the drug is discontinued abruptly. In general, the prognosis for treatment of epilepsy is good. About $70 \%$ of patients' present seizure control for approximately five years, and $50 \%$ manage to stay medication 
free without recurrence of seizures ${ }^{4}$. Following is a summary of the pharmacology of the major anticonvulsants, with emphasis on the indication, mechanism of action, adverse effects and drug interactions.

Phenobarbital: long-term effect barbiturate, effective against all types of epilepsy, but not the primary generalized non-convulsive type. Despite the low cost, presents cognitive and behavioral effects that limit the use of this drug to treat epilepsy; considered second-line treatment of epilepsy $1,10,15$. Phenobarbital exerts its antiepileptic effect by modulating the postsynaptic action of gamma aminobutyric acid (GABA), an inhibitory neurotransmitter, and also by the block of postsynaptic excitatory effect induced by glutamate, especially those mediated by the activation of the AMPA ( $\alpha$-amino-3-hydroxy5-methyl-4-isoxazolepropionic acid) receptor. . The GABAergic action causes increased duration of chloride channels opening, promoting neuronal hyperpolarization, limiting activity and increasing the seizure threshold ${ }^{1,15,16}$. The main adverse effects include sedation, depression, hyperactivity (in children), confusion (in elderly), skin changes, megaloblastic anemia, osteomalacia, nystagmus, ataxia, abnormal collagen deposition (Dupuytren's contracture), dependence, tolerance and low therapeutic index 4-5,8,10,16,25. The drug, like other barbiturates, may precipitate attacks of porphyria ${ }^{10}$. Congenital defects can occur when phenobarbital is chronically administered during pregnancy. Changes in coagulation and bleeding in the neonate can occur. Interaction of phenobarbital with other drugs arises from the hepatic microsomal enzyme induction by phenobarbital, which may persist for 30 days after discontinuation of the drug. This can exacerbate the hepatic metabolism of many liposoluble drugs, such as anticonvulsants, oral contraceptives, beta-blockers, corticosteroids, digoxin, anti-inflammatory drugs and anticoagulants ${ }^{10,15,17}$. The enzyme induction caused by phenobarbital may increase the biotransformation and toxicity of inhaled anesthetics. Therefore isoflurane should be the drug of choice ${ }^{17}$.

Valproic acid: it is a carboxylic acid (2-propylpentanoic acid) effective in all generalized primary epilepsy and all convulsive epilepsy types. It is less effective in the treatment of partial non convulsive epilepsy ${ }^{1,15}$. The valproic acid is a weak inhibitor of two enzymatic systems that inactivate GABA: the GABA transaminase and succinate semialdehyde dehydrogenase. There is some evidence that the drug may potentiate the action of GABA by a postsynaptic action ${ }^{10}$. This drug also works by sustained and repetitively limiting the neuronal discharge through voltage-dependent sodium channels, that is, it prevents the generation of action potential bursts that constitute the microphysiological substrate of the epileptic neuron ${ }^{1,15}$. Drug adverse effects include tremors, weight gain, dyspepsia, nausea and vomiting, anorexia, alopecia, edema, encephalopathy (due to increased levels of ammonia), teratogenicity, agranulocytosis, aplastic anemia, allergic dermatitis, Stevens-Johnson syndrome, hepatotoxicity (especially in children younger than 2 years of age), platelet changes that can lead to bleeding, and pancreatitis $4,8,10,15$. Valproic acid and phenytoin may displace diazepam from its protein binding sites, resulting in increased pharmacological activity of these drugs. Furthermore, it is an enzyme inhibitor, leading to a decrease in metabolic rate of phenytoin. With chronic use of this drug, plasma concentration of phenobarbital increases by approximately 50\%, probably due to inhibition of liver microsome enzymes ${ }^{15}$.

Felbamate: normally used to treat adults presenting isolated partial epilepsy or secondary generalized epilepsy. In children, it is used in the treatment of partial or generalized epilepsy associated with Lennox-Gastaut syndrome ${ }^{10,15}$. The mechanism of action, although not fully explained, appears to be related to the decrease of sodium ion current (block of voltage-dependent sodium channels), increased the inhibitory effects of GABA, and blockage of the N-methyl-D-aspartate receptors ${ }^{15}$. The adverse effects described are insomnia, anorexia, nausea, headache, irritability, aplastic anemia and hepatotoxic effects 4,15,10. Monitoring through blood and liver function tests is indicated ${ }^{15}$. Felbamate interacts with other anticonvulsants ${ }^{10}$. Should the drug be used in combination with phenytoin, carbamazepine or valproic acid, the drug dosage should be reduced between $20 \%$ and $30 \%$ in order to prevent toxic effects ${ }^{15}$.

Phenytoin: it is effective in the treatment of partial and generalized epilepsies ${ }^{10,15}$. It has a high therapeutic index. Phenytoin regulates neuronal excitability and thus the spread of seizure activity from the seizure focus by blocking voltage-dependent sodium channels, possibly in the transport of calcium through the neuronal membrane ${ }^{10,15,16}$. The stabilizing effect on the cell membrane is relatively selective for the cerebral cortex, but also extends to peripheral nerves. Additionally to the effect on the ion flow, phenytoin acts on the second messenger systems as calmodulin and cyclic nucleotides ${ }^{15}$. Side effects of phenytoin include nystagmus, diplopia, dizziness (vestibular cerebellar dysfunction), ataxia, nausea and vomiting, gingival hyperplasia, depression, megaloblastic anemia, drowsiness, agranulocytosis, aplastic anemia, allergic dermatitis, Stevens-Johnson syndrome, hyperglycemia, hepatotoxicity, pancreatitis, acne, rough skin, hirsutism, teratogenicity and Dupuytren's contracture 4,8,10,15,16. Chronically administered during pregnancy, the drug can cause congenital malformations ${ }^{15}$. Phenytoin can induce the oxidative metabolism of several liposoluble drugs such as carbamazepine, valproic acid, ethosuximide, corticosteroids and anticoagulants ${ }^{15,16}$. Patients treated with this drug have decreased plasma concentrations of thiopental, propofol, midazolam, opioids and neuromuscular non depolarizing blockers ${ }^{8,17}$.

Phosphofenitoin: it is a prodrug of phenytoin, presenting the same pharmacological properties. Its main indication is the replacement of intravenous phenytoin in the treatment of status epilepticus ${ }^{18}$.

Carbamazepine: is used in the treatment of convulsive and nonconvulsive partial epilepsy, as well as trigeminal and glossopharyngeal nerves neuralgia, bipolar disorders and alcohol withdrawal syndrome ${ }^{10,15}$. Carbamazepine changes the ionic conductance to sodium with a membrane stabilizing effect ${ }^{15,16}$. Its side effects can be sedation, diplopia, dizziness, neutropenia, nausea, drowsiness, diarrhea, jaundice, oliguria, hypertension, cardiac arrhythmias, hyponatremia, agranulocytosis, aplastic anemia, allergic dermatitis, Stevens-Johnson 
syndrome and systemic lupus erythematosus (SLE) 4,8,10,15,16. In addition to accelerating its own metabolism, carbamazepine exacerbates hepatic oxidation and conjugation of other liposoluble drugs. The most common interactions occur with oral contraceptives, haloperidol, valproic acid, theophylline, ethosuximide, clonazepam, phenobarbital, steroids, anticoagulants and antipsychotic drugs 10,15,16. Drugs that inhibit the metabolism of carbamazepine may increase its toxicity and include cimetidine, propoxyphene, diltiazem, verapamil, erythromycin and isoniazid 10,15,16. Due to its potent enzyme inducer, carbamazepine reduces the plasma concentrations of thiopental, propofol, midazolam, opioids and neuromuscular non depolarizing blockers ${ }^{10,17}$. There is risk of hepatotoxicity after anesthesia with halothane, enflurane, and possibly with sevoflurane ${ }^{17}$.

Oxcarbazepine: it was designed to prevent the formation of the epoxide metabolite of carbamazepine, responsible for many toxic effects of the drug ${ }^{10}$. It has blocking action of the voltage-dependent sodium channels. It is generally better tolerated than carbamazepine. The most common adverse effects are sedation, dizziness, headache, amnesia, ataxia, diplopia, depression, insomnia, anxiety, nausea and vomiting, diarrhea, hyponatremia, acne, alopecia and urticaria 8,10 . It shows less pharmacokinetic changes, not inducing the metabolism of other antiepileptic drugs ${ }^{10}$.

Lamotrigine: it acts by stabilizing the voltage-sensitive sodium channels and preventing the release of glutamate and aspartate $10,15,16$. This drug has a broad spectrum of activity and it is effective when used alone or in combination in adult patients with partial or generalized epilepsy, and in children with Lennox-Gastaut syndrome ${ }^{10,15}$. It shows, as side effects, dizziness, diplopia, tremor, insomnia, aggression, ataxia, allergic dermatitis, Stevens-Johnson syndrome, headache, nausea, vomiting and blood dyscrasias 4,8,10,15,16. Lamotrigine induces the hepatic microsomal system, speeding up their metabolism. The valproic acid slows the metabolism of lamotrigine, increasing its elimination half-life ${ }^{15}$.

Gabapentin: it is used in the treatment of partial epilepsy and generalized partial seizures and in treating various pain syndromes, such as erythromelalgia, reflex sympathetic dystrophy and neuropathic pain ${ }^{15}$. The mechanism of action is unclear, appearing to act by binding to specific receptors in the brain, inhibiting the voltage-dependent sodium current, apart from also increasing GABA action ${ }^{4,15}$. The main adverse drug effects are sedation, diplopia, dizziness, ataxia, skin changes, weight gain, nausea and leucopenia 8,10,15.

Primidone: it is metabolized in phenobarbital and another active metabolite, phenylethylmalonamide. The effectiveness of the drug is similar to phenobarbital, but less tolerated 4,10,15,16. Adverse reactions include fatigue, depression, psychosis, decreased libido, skin changes, leukopenia, thrombocytopenia and SLE ${ }^{8,10}$. Chronic use of the drug increases the metabolism of enflurane, halothane, and probably isoflurane ${ }^{17}$.

Clonazepam: in general, it is associated with other drugs. It is the drug of choice only in myoclonic seizures ${ }^{15}$. It binds to $\mathrm{GABA}_{A}$ in the brain, enhancing the inhibitory transmission mediated by GABA, which increases the conductance to chloride ion, due to an increase in frequency of chloride channels' opening, leading to cell hyperpolarization and inhibition of neuronal trigger 4,15,16. The adverse effects of the drug include personality changes manifested as behavioral disorders, including hyperactivity, irritability and difficulty concentrating, especially in children. Depression can occur in the elderly. Sialorrhea and increased bronchial secretion may be common in children. Generalized convulsive activity may occur if the drug is abruptly discontinued $8,15,16$.

Ethosuximide: it is the drug of choice in treating absence seizures (petit mal). It acts by decreasing conductance in voltage-dependent calcium channels in thalamic neurons 10,15,16. Adverse effects of the drug include nausea, anorexia, vomiting, lethargy, agitation, headache, drowsiness, dizziness, ataxia, photophobia, agranulocytosis, aplastic anemia, allergic dermatitis, Stevens-Johnson syndrome, SLE and, rarely, bone marrow depression 8,10,15,16.

Topiramate: it is an antiepileptic drug used initially as an adjuvant to partial seizures ${ }^{10}$. Additional studies suggest its efficacy as monotherapy and also in the tonic-clonic seizures and in Lennox-Gastaut syndrome ${ }^{10}$. The drug acts by multiple mechanisms, such as blocking a subtype of non-NMDA glutamate receptor, potentiating the action of GABA and blocking sodium channels $4,10,16$. Side effects include dizziness, nervousness, cognitive disturbances, ataxia, depression, diarrhea, glaucoma, loss of appetite and weight loss, paresthesias and development of urolithiasis $4,8,10,16$. Patients treated with topiramate should be evaluated under clinical and laboratory aspects, preoperative assessment to detect asymptomatic metabolic acidosis, which can occur with chronic use of the drug ${ }^{19}$.

Levetiracetam: it is used in the treatment of partial seizures as adjunctive or monotherapy ${ }^{10}$. It is generally well tolerated. The mechanism of action of the drug has not been determined, although recent studies show clearly that the drug binds to protein A2 (SV2A), related to the release of glutamate in the synaptic vesicle ${ }^{16,20}$. Common side effects include drowsiness, weakness, dizziness, ataxia, amnesia, depression, anxiety, anorexia, diarrhea, dyspepsia, skin changes and pancytopenia ${ }^{8,10}$. It presents little risk of drug interactions ${ }^{10}$.

Tiagabine: it is indicated in adjuvant therapy of patients with partial seizures. Clinical trials indicate drug efficacy in tonic-clonic seizure when combined with other anticonvulsants ${ }^{10}$. The action mechanism of the drug is the blockage of GABA reuptake ${ }^{10,16}$. The most frequent side effects are dizziness, asthenia, somnolence, anxiety, nausea, nervousness, tremors, abdominal pain and cognitive disorders 8,10,16.

Zonisamide: it is an antiepileptic drug used in the broad spectrum of adjuvant therapy of partial seizures and secondarily generalized seizures. It is also shown to be effective in controlling the generalized tonic-clonic epilepsy, the myoclonic epilepsy and Lennox-Gastaut syndrome. It has multiple mechanisms of action. It has its pharmacological effect by blocking voltage-dependent sodium channels, also inhibiting the calcium current through T-type channels. The drug has an effect on the synthesis, degradation and release of several neurotransmitters such as glutamate, GABA, dopamine, sero- 
tonin and acetylcholine, which increases synaptic inhibition. It is a carbonic anhydrase inhibitor, although this effect is not correlated with epileptic activity. Adverse effects include sedation, asthenia, diplopia, hypohidrosis, skin changes, StevensJohnson syndrome, dizziness, headache, ataxia, anorexia, agitation, irritability, and nephrolithiasis ${ }^{21}$. It may cause metabolic acidosis. Serum bicarbonate levels should be evaluated before treatment and periodically after it, even in the absence of symptoms 22 .

\section{OTHER THERAPIES}

$\mathrm{ACTH}$ and corticosteroids: in children with West syndrome, the use of ACTH and corticosteroids is well established. The mechanism of action is uncertain, postulating effects on cholinergic modulation or serotoninergic ${ }^{23}$. It is important that the anesthesiologist knows the adverse effects of corticosteroids that can influence the conduct of anesthesia and involve multiple organ systems. These effects include irritability, Cushing syndrome, electrolyte disturbances (hypokalemia), impaired glucose tolerance, dilation of cerebral ventricles, increased cerebrospinal fluid, hypertension, cardiomyopathy, and sepsis 4,23 .

Ketogenic diet: an alternative therapy for refractory epilepsy in children and adolescents is the ketogenic diet, which consists of a diet rich in fats and low in carbohydrates and proteins, leading to ketosis and production of beta-hydroxybutyrate, which could have an antiepileptic effect, probably due to the increase in brain energy reserves ${ }^{4,23,24}$. This diet seems to work better in generalized seizures (absence seizures and myoclonus), but any type of crisis may benefit from this kind of therapy ${ }^{23}$. Ketosis leads to metabolic acidosis, which can be exacerbated by products that contain carbohydrates. It is important to avoid solutions in pre-medication containing glucose, and dextrose-containing intravenous solutions, which can cause a decrease in plasma levels of ketones and increased risk of seizures ${ }^{4}$. Children with ketogenic diet can safely undergo general anesthesia. Although serum glucose levels generally do not change, the $\mathrm{pH}$ and serum bicarbonate levels should be monitored every 2 or 3 hours, even on short procedures to avoid severe metabolic acidosis ${ }^{4,25}$.

\section{SURGICAL TREATMENT}

Surgical treatment is indicated in cases of refractory epilepsy with drug treatment. Surgical treatment includes frontal lobectomy, focal cortical resection, Calostoma (section on the bridge level through which the fibers that connect one brain hemisphere to the other), hemispherectomy and placement of the vagus nerve stimulator ${ }^{26}$.

The vagus nerve stimulator consists of a bipolar wire with two electrodes involving the left vagus nerve at cervical level, and a generator implanted subcutaneously in the infraclavicular region ${ }^{26,27}$. Laboratory studies suggest that this device increases the release of noradrenaline in the locus coeruleus, increasing the seizure threshold. The most common adverse effects with the use of such equipment are voice changes and pain in the pharynx. In patients with the vagus nerve stimulator undergoing surgery, the equipment must be evaluated before and after surgery. It is important to emphasize that these patients are at risk of aspiration because of the possibility of laryngeal-pharyngeal dysfunction during vagus nerve stimulation. Although there are no reports of electromagnetic interference with the use of electrocautery and external defibrillator, these devices can damage the electrodes and the generator of the vagus nerve stimulator. Similar care should be taken with patients with cardiac pacemakers. During activation of the vagus nerve stimulator in patients with obstructive sleep apnea, airway obstruction can occur. Considering the association of the residual effect of anesthesia and the activation of the vagus nerve stimulator, airway obstruction in the postoperative period in patients with obstructive sleep apnea may accur. Central venous access on the side of stimulation of the vagus nerve should be avoided. The neck should be placed in neutral position in order to avoid stretching or displacement of the electrodes ${ }^{28}$.

\section{ANESTHETIC APPROACHES IN EPILEPTIC PATIENT}

\section{Anesthetic drugs and epilepsy}

Chloral hydrate: this drug is used to induce sleep during the EEG, and to promote sedation in preanesthetic medication in children. It has shown anticonvulsant properties ${ }^{4,29}$. Through oral administration, the drug latency to induce sleep is 40 minutes in average, while for sedation, the onset is unpredictable, ranging from 30 to 60 minutes. It is considered a safe drug, although reports of deaths and severe neurological injury have been reported ${ }^{30}$. There is a risk of respiratory depression and hypoxemia, especially in patients at risk for airway obstruction, for example, Down syndrome, or obstructive sleep apnea after tonsillectomy. Other adverse effects include nausea, vomiting, restlessness, sweating, dizziness and sedation failure 4,29,31,32.

Benzodiazepines: they have anticonvulsant activity. However, benzodiazepines can cause short periods of seizure activity, and in EEG in patients with Lennox-Gastaut syndrome, a form of epilepsy of difficult treatment in children $8,33,34$. Benzodiazepines are commonly used to treat acute seizures ${ }^{4}$. The antiepileptic effect of benzodiazepines is due to its potentiating action of the inhibitory transmission mediated by $\mathrm{GABA}_{A}$, increasing the opening frequency of chloride channels and leading to neuronal hyperpolarization ${ }^{8,33}$. Midazolam has potent anticonvulsant activity and is commonly used in the treatment of status epilepticus mainly in children 8,35,36. As noted, clonazepam is effective in the prevention and treatment of seizures, especially in myoclonic seizures in children. Some antiepileptic drugs have significant sedative effects, such as phenobarbital and carbamazepine, which have the ability to potentiate the effects of anesthetics and other central nervous system depressants ${ }^{8,37}$. 
Flumazenil may precipitate seizures in patients treated with benzodiazepines or who received this drug as part of premedication due to its competitive antagonism ${ }^{38,39}$. It has been reported that the use of flumazenil to reverse benzodiazepine sedation caused generalized convulsions and coma in epileptic patients who underwent the procedure under local anesthesia and sedation with diazepam ${ }^{40}$. Given its proconvulsant effects, flumazenil should be used with caution in patients with epilepsy ${ }^{41}$.

Propofol: in spite of the conflicting results in the literature, there is clinical evidence that propofol has anticonvulsant effect and is considered a safe drug for sedation, induction and maintenance of general anesthesia in children and adults $4,8,33,34,42$. In patients with epilepsy, the occurrence of seizures of epileptic origin is extremely rare with the use of propofol, and occurs frequently in the recovery from anesthesia ${ }^{42}$. The antiepileptic activity of propofol is probably related to inhibition of pre-and postsynaptic chloride channels mediated by GABA ${ }^{8}$. Propofol can cause abnormal movements, as opisthotonus and myoclonia in both epileptic and healthy patients, but these changes do not seem to relate to epileptogenic activity ${ }^{43-45}$. Propofol is an effective alternative in the treatment of refractory epileptic seizures to usualantiepileptic drugs and in cases of status epilepticus 46-48.

Etomidate: etomidate appears to have anticonvulsant activity at high doses ${ }^{33.49}$ and pro-convulsant characteristics in usual clinical doses $33,50-54$. Threfore, it is recommended to avoid its use in epileptic patients $4,8,34$.

Ketamine: although there are reports of anticonvulsant property of ketamine, especially when associated with drugs with GABAergic activity (benzodiazepines and propofol) for cases refractory to usual therapy ${ }^{33,55-59}$, most of the clinical evidence shows that ketamine, at usual doses, has significant epileptogenic potential ${ }^{33,54,60}$, and should be, therefore, avoided in epileptic patients $4,8,34,61$.

Droperidol: it does not produce neuron excitability nor induce seizures in epileptics; it can be used safely in these patients 4,54 .

Dexmedetomidine: sedation with dexmedetomidine observed in the EEG shows a pattern similar to stage II sleep. It presents no pro- or anticonvulsant effects and has been used successfully in children with autism undergoing EEG ${ }^{4,62}$. This drug has been safely used in adult patients for epilepsy surgery, allowing an adequate mapping of epileptic foci and subsequent resection ${ }^{63,64}$. Thus, dexmedetomidine can be used safely in the epileptic patient.

Clonidine: the effect of clonidine in epileptic patients is controversial. There are reports that the drug lowers the seizure threshold in epileptic patients (pro-convulsant effect) ${ }^{65-67}$. Other authors have used the drug in autistic patients (epilepsy in $50 \%$ of patients), with the purpose of sedation for the performance of EEG, and concluded that there was no effect on the EEG. Moreover, it is easy to be administered, the sedation time is short and it promotes good quality sedation ${ }^{68}$. In animal experiments, clonidine presented no pro- or anticonvulsant activity in the lidocaine-induced seizures ${ }^{69}$. So there is not enough clinical evidence at present that the use of clonidine is contraindicated in epilepsy patients.
Barbiturates: barbiturates, except for the methohexital, present a significant anticonvulsant activity in the EEG ${ }^{4}$, and its use is considered safe for induction of anesthesia in epileptic patients, as well as treatment of status epilepticus ${ }^{8,34}$.

Opioids: meperidine has neuroexcitatory effect attributed to its metabolite normeperidine. Clinically, it is characterized as tremors, myoclonus and seizures ${ }^{8,34,70}$. At the doses commonly used as adjuvant drug in general or regional anesthesia, it appears safe in the epileptic patient. However, in situations where there may be accumulation of normeperidine, such as prolonged use (patient-controlled analgesia), anticonvulsants (phenytoin and phenobarbital) and phenothiazines (chlorpromazine), patients with renal failure, advanced malignancy and sickle cell disease, the drug can cause seizures; it shall then not be used in epileptic patients ${ }^{70}$.

Morphine has no pro-convulsant effect when used intravenously and can be used safely in epileptic patients. However, tonic-clonic seizures were observed when employing epidural drug in epileptic patients 8,70 .

Regarding phenylpiperidine derivatives (fentanyl, alfentanil, remifentanil and sufentanil), there are several reports that the administration of these drugs cause tonic-clonic movements, although in many of these articles there have been no EEG monitoring, making it difficult to confirm whether these movements are the result of seizure activity or whether, in fact, represent myoclonus or stiffness of skeletal muscles 57,70 . In other studies with EEG monitoring, there was no correlation between these movements and tonic-clonic seizure activity ${ }^{70}$. However, there are reports of epileptogenic EEG activity with the use of fentanyl, alfentanil and remifentanil - these drugs can be helpful in localizing the epileptic focus ${ }^{71-73}$. Therefore, we recommend avoiding the use of high doses or rapid administration of opioids of the phenylpiperidine group in epileptic patients ${ }^{8}$. It is important to emphasize that there may be pharmacokinetic interaction between the phenylpiperidine opioids (mostly fentanyl) and anticonvulsant drugs, leading to a greater need to administer opioids during anesthesia ${ }^{74}$.

Nitrous oxide: although nitrous oxide has excitatory effects on central nervous system, the epileptogenic potential of the drug is very low and can be used safely in epileptic patients $4,8,34,70$.

Halothane: most published studies show that halothane has potent anticonvulsant effect, and can be used safely in epileptic patients. However, there are reports of seizures associated with halothane ${ }^{34,75,76}$.

Enflurane: it may produce epileptiform EEG activity and seizures, especially at concentrations above 2 MAC and hypocapnia in the presence of stimulation, tactile, visual or hearing impairment; then it should be avoided in epileptic patients $8,34,70,75,77,78$.

Isoflurane: although there are reports of potential epileptogenic with isoflurane ${ }^{79}$, most studies show potent anticonvulsant effect of the drug, that is also used in the treatment of status epilepticus ${ }^{80}$, and certainly in epileptic patients 4,8,34,70,75. 
Sevoflurane: the epileptiform activity of sevoflurane has been extensively studied ${ }^{57}$. A review of 30 studies evaluated the correlation between the use of sevoflurane and epileptiform EEG changes and/or presence of convulsive movements in patients with or without epilepsy. The incidence of these changes ranged from $0-100 \%$. The depth of anesthesia with sevoflurane and/or the presence of hyperventilation (hypocapnia) were risk factors for the emergence of excitatory activity of the central nervous system. The authors of this review recommend avoiding this drug at concentrations above 1.5 MAC in epileptic patients and in the presence of hypocapnia ${ }^{81}$.

Desflurane: although there are few studies, there is no evidence that desflurane has epileptiform activity, even when used in high concentrations or in the presence of hypocapnia, and has even been administered in the treatment of status asthmaticus and safely in epileptic patients $8,82,83$.

\section{Neuromuscular blockers}

Some antiepileptic drugs have a depressant action on acetylcholine release at the neuromuscular junction ${ }^{8}$. Phenytoin and carbamazebina, when used chronically, decrease the duration of action of some neuromuscular blockers such as rocuronium ${ }^{84,85}$, pancuronium ${ }^{86}$, vecuronium ${ }^{87}$, cisatracurium ${ }^{88}$, due to increased hepatic metabolism of these drugs. In contrast, atracurium ${ }^{89,90}$ and mivacurium ${ }^{91}$ that are not dependent on the hepatic metabolism for their elimination, have not presented reduction in the time of action. Acute administration of phenytoin potentiates the neuromuscular blockade of rocuronium, by an unknown mechanism ${ }^{92}$. Although laudanosine, an atracurium metabolite, may present epileptiform EEG activity and seizures when administered in higher concentrations ${ }^{93}$ in experimental animals, it is unlikely that those effects can be observed with the usual doses used in anesthesia. With regard to succinylcholine, there is small increase in duration of action in patients chronically treated with antiepileptic drugs, factor which is irrelevant under the clinical point of view ${ }^{94}$. Because of drug interactions between antiepileptic drugs and neuromuscular blockers, we recommend intraoperative monitoring of neuromuscular blockade ${ }^{8}$.

Anticholinesterase agents: it has not been observed epileptiform activity on EEG or seizures after administration of cholinesterase inhibitors during anesthesia in patients with epilepsy 8,34 .

Anticholinergics: atropine, scopolamine and glycopyrrolate can be used safely in the epileptic patient ${ }^{8}$.

Local Anesthetics: local anesthetics have pro-convulsant and anticonvulsant properties due to the stabilizing effect of the membrane. In small doses, local anesthetics reduce cerebral blood flow and metabolism, as well as brain electrical activity, and act as anticonvulsants, sedatives and analgesics, while at high doses it act as pro-convulsant drug, lowering the seizure threshold in the cerebral cortex, amygdala and hippocampus, leading to generalized convulsions ${ }^{34,95}$. The systemic toxicity associated with regional anesthesia is a cause of seizures in approximately $5 / 10.000$ patients, which can be found even with local anesthetics for use later. It is more frequent with bupivacaine and those regional anesthesia techniques in which large doses of local anesthetics such as epidural and caudal are employed 8,95-97.

A retrospective study on 335 patients with different types of epilepsy undergoing regional anesthesia showed that 24 patients (6\%) had seizures in the perioperative period. In 19 patients, seizures were the result of epileptic disease and not related to the use of local anesthetic. In the 5 remaining patients, systemic toxicity resulting from the use of local anesthetic cannot be ruled out. An interesting aspect of the study is that the seizures were more frequent in those patients who had presented with epileptic seizures less than a week prior to surgery and used phenytoin as an anticonvulsant drug. As the seizure threshold appears to be decreased in patients with recent seizures, it is important to be prepared to treat any seizure in these patients when using local anesthetics in the perioperative period ${ }^{98}$.

\section{ANESTHETIC MANAGEMENT IN THE EPILEPTIC PATIENT}

\section{Preoperative assessment and premedication}

In the perioperative management of epileptic patients, it is important, whenever possible, an adequate control of the disease, being essential a careful review of medical history, especially in regard to the evolution of the disease, factors triggering the seizures (fasting, stress, sleep deprivation, alcohol and drugs), and comorbidities and their treatment ${ }^{4,8}$. The presence of mental retardation, hypotonia, and risk factors for aspiration and airway obstruction should be examined 4 .

A preoperative evaluation of the neurologist responsible for the patient is recommended, especially in the case of recent changes in disease evolution ${ }^{8,57}$.

Anticonvulsants should be used until the day of surgery, including in children and pregnant women 8 .

It is important to know the adverse effects of anticonvulsants, as well as the possibility of drug interactions with anesthetic agents ${ }^{4}$.

The plasma dosing of antiepileptic drugs should not be done in a systematic way, unless there have been recent changes in drug dosage or in the case of onset of kidney or liver failure, arrhythmias and electrolyte changes ${ }^{8}$.

The premedication is usually carried out with the use of a benzodiazepine, midazolam being the most widely used due to its potent anticonvulsant and anxiolytic effects. It is important to emphasize that some anticonvulsants, and the ketogenic diet can cause sedation and interact with benzodiazepines.

\section{CONDUCT DURING SURGERY}

The monitoring of epileptic patients is based on the type of surgical procedure being performed and the patient's clinical 
condition. Patients undergoing major surgical procedures or severe clinical conditions require invasive monitoring. In most procedures, the basic monitoring is adequate.

The literature shows that most beta-lactam antibiotics showed some degree of pro-convulsant activity in laboratory animals and in clinical trials, probably due to inhibition of inhibitory postsynaptic response mediated by $\mathrm{GABA}_{A}{ }^{99}$. However, the widespread use of these drugs in epileptic patients in the perioperative period, with no reports of epileptogenic activity, seems to show that these antibiotics can be safely used in patients with epilepsy.

On inducing anesthesia in epileptic patients, the most commonly used drugs are thiopental, benzodiazepines and, propofol despite its pro-and anticonvulsant effects. The use of ketamine and etomidate should be avoided 8,57 .

During maintenance of anesthesia, the inhalational agent most often used due to its potent anticonvulsant effect is isoflurane. Sevoflurane administered at concentrations of less than 1.5 MAC (avoiding hyperventilation) seems safe in the epileptic patient. Halothane and desflurane may also be used safely. The use of nitrous oxide is controversial, and enflurane is contraindicated in those patients 8,57 .

Although there are reports of convulsive movements with the use of opioids, these drugs have low seizure frequency when used in low doses and slow intravenous administration. Meperidine can cause seizures through its metabolite normeperidine, and should be avoided in epileptic patients. The use of neuraxial opioids rarely cause seizures, which leads to no contraindication to its use in the epileptic patient ${ }^{8}$.

In the intraoperative handling of the epileptic patient, it is important to avoid changes that reduce the seizure threshold, such as hypoxia, hypotension, hypocapnia, and hyponatremia ${ }^{28}$.

The regional anesthesia techniques can be used safely in the epileptic patient. However, coagulation changes that may occur with the administration of most antiepileptic drugs should be evaluated ${ }^{8}$.

\section{POSTOPERATIVE MANAGEMENT}

Anticonvulsant drugs should be restarted as soon as possible after surgery, according to the time of fasting. If the fasting time is 12-24 hours, there is no need to replace the oral by the parenteral route, and treatment should be restarted when the oral route is cleared. In the case of fasting over 24 hours and in the chronic use of the orally administered anticonvulsant drugs phenytoin or phenobarbital, we replace the enteral route for venous 2-3 times a day ${ }^{8}$. When there is no presentation for parenteral use with anticonvulsant drugs used orally by the patient, it is possible to replace these drugs by temporarily $3 \mathrm{mg} \cdot \mathrm{kg}^{-1}$. dia ${ }^{-1}$ phenytoin or intravenous phenobarbital $2.3 \mathrm{mg} \cdot \mathrm{kg}^{-1} \cdot \mathrm{dia}^{-1}$ in 2-3 doses ${ }^{8}$.

After surgery, whenever possible, the monitoring of plasma levels of antiepileptic drugs for at least 48 hours should be performed because there are significant variations in the apparent volume of distribution, linked to plasma proteins, hepatic metabolism and renal elimination 8,100 .

\section{PERIOPERATIVE SEIZURES IN EPILEPTIC PATIENTS}

The seizures of epileptic origin are rare in the perioperative period, usually occurring in the induction and recovery from anesthesia. However, they can happen up to 72 hours after surgery ${ }^{57}$.

It is important to remember that the anesthetic drugs which commonly induce epileptogenic activity rarely progress to seizures in the perioperative period ${ }^{57}$, although there is greater propensity to occur more frequently in epileptic patients than in non-epileptic patients ${ }^{57}$.

The effects of general anesthesia were assessed in 236 epileptic patients of both sexes with an average age of 21 years (range 1 month to 84 years) undergoing diagnostic and therapeutic procedures, excluding neurosurgical procedures and invasive neurological examinations. The procedures performed included magnetic resonance imaging (35.7\%), surgical procedures (32.1\%), other imaging studies of non-nuclear magnetic resonance (16.9\%), biopsies (12.1\%), and dental procedures (3\%). Partial epilepsy was present in $70.7 \%$ of patients, generalized epilepsy in $27.1 \%$, and epilepsy of unknown cause in $2.1 \%$. A total of 297 procedures were evaluated under anesthesia. Ten procedures were performed under local or regional anesthesia, a procedure under spinal anesthesia and the other under general anesthesia. Seizures were observed in $6(2 \%)$ patients, and therapy with intravenous benzodiazepine was only necessary in one patient.

The seizure activity was observed in the induction of anesthesia $(n=1)$ or after the procedure before recovery from general anesthesia $(n=5)$. Five of the patients who had seizures were younger than 18 years (mean $=8$ years ranging from 1 to 13 years). Five of the patients who had had epileptic seizures had refractory epilepsy, requiring multiple anticonvulsant drugs. The drugs used in general anesthesia of patients included sevoflurane, isoflurane, nitrous oxide, propofol, thiopental, ketamine, fentanyl, atracurium, cisatracurium and vecuronium. None of the patients who received local anesthesia, regional or spinal anesthesia showed seizure activity. This article shows that the incidence of perioperative seizures in epileptic patients is low and does not require anticonvulsant therapy in most patients. It is most common in children and in patients with refractory epilepsy that require multiple anticonvulsant drugs ${ }^{101}$.

Although the occurrence of seizures in the perioperative period is low, they can be serious, if prolonged, leading to brain damage from hypoxia, apnea, prolonged postoperative mechanical ventilation, and delayed awakening from anesthesia. The seizure activity may impair the physiological regulation of cardiac and respiratory activities. Although the seizures are associated with tachycardia and tachypnea, there are reports of apnea and bradycardia following the seizure, and leading to sudden death in epileptic patients, probably due to autonomic instability, which causes cardiac arrhythmias or neurogenic pulmonary edema ${ }^{101,102}$.

In the case of the emergence of seizures in the perioperatory period, the initial approach is to maintain a patent airway with adequate ventilation and protect the patient from injuries resulting from seizures. Monitoring should be performed us- 
ing cardioscope, blood pressure and pulse oximetry. Seizures lasting less than five minutes do not usually require treatment 4,8 .

Should convulsions persist for more than five minutes, intravenous benzodiazepine should be used. The preferred drug is lorazepam, which, however, is not available for parenteral use in Brazil. In our country, the best alternative is diazepam (5-20 mg). Should convulsions persist, a second dose of benzodiazepine associated with phenytoin $\left(20 \mathrm{mg} \cdot \mathrm{kg}^{-1}\right.$, 30 minutes) should be used. In the case of refractory seizures, phenobarbital (1.5 mg. $\mathrm{kg}^{-1} \cdot \mathrm{min}^{-1}$ or $100 \mathrm{mg} / 70 \mathrm{~kg} \cdot \mathrm{min}^{-1}$ can be used with a maximum dose of $15 \mathrm{mg}^{\mathrm{kg}} \mathrm{k}^{-1}$ or $1,000 \mathrm{mg} / 70 \mathrm{~kg}$ ), midazolam (0.1-0.3 mg. $\mathrm{kg}^{-1}$ in 2 to 5 minutes, followed by infusion of 0.05 to $0.4 \mathrm{mg} \cdot \mathrm{kg}^{-1}$.hour $\left.{ }^{-1}\right)$, propofol (1-2 mg. kg-1 followed infusion of $\left.2-10 \mathrm{mg} \cdot \mathrm{kg}^{-1} \cdot \mathrm{h}^{-1}\right)$, thiopental $\left(5-10 \mathrm{mg} \cdot \mathrm{kg}^{-1}\right.$ in 10 minutes, followed by infusion of $100-400 \mathrm{mg}^{-1} \mathrm{~h}^{-1}$, lidocaine (1.5-2 mg. $\mathrm{kg}^{-1}$ in 2-5 minutes, followed by infusion of 2-3 $\mathrm{mg} \cdot \mathrm{kg}^{-1} \cdot \mathrm{h}^{-1}$ for 12 hours), isoflurane ( 0.5 to $1.5 \%$ ) and ketamine (50-100 mg followed by infusion of 50-100 mg.h $\left.{ }^{-1}\right)^{8,57,103}$.

In summary, in the perioperative management of epileptic patients, it is important for the anesthesiologist in the preoperative evaluation to identify the type of epilepsy, frequency, intensity and the triggers to the epileptogenic episodes, the use of anticonvulsant drugs and possible interactions with drugs used in anesthesia as well as the presence of other treatments such as vagus nerve stimulator and ketogenic diet and its implications in anesthetic techniques. The anesthesiologist must know the properties of pro- and anticonvulsant drugs used in anesthesia, minimizing the risk of seizure activity during surgery. Finally, although an uncommon event, it is important to outline the diagnosis and, where necessary, establish the treatment of perioperative seizures, which would allow lower morbidity and mortality in patients with epilepsy. 